\section{From industry to academia}

Growing recognition of the importance of translational research is providing new opportunities to apply expertise gained in industry in academic institutions. This month, we feature two researchers who have recently moved from pharma to academia.

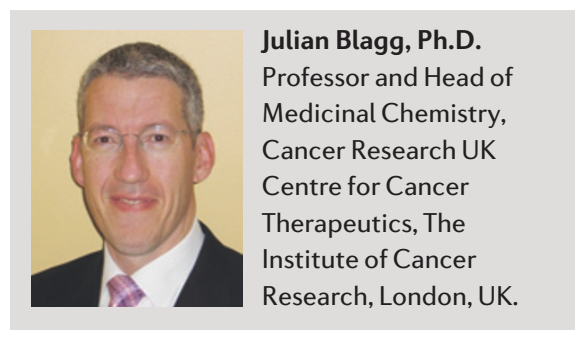

In April 1994, The Institute of Cancer Research - one of Europe's leading centres in cancer research - together with Cancer Research UK set up the Centre for Cancer Therapeutics with the aim of speeding the translation of advances in cancer biology into potential drugs. Julian Blagg, previously Executive Director of Exploratory Medicinal Sciences at Pfizer in Sandwich, UK, has very recently taken over as Head of Medicinal Chemistry for the centre - a position that involves leading a team of 50 chemists designing, synthesizing and developing potential anticancer drugs in collaboration with colleagues in biology, pharmacokinetics and clinical research.

"After 19 years in the pharmaceutical industry, I wanted to experience and contribute to a different drug discovery model in a disease area I care passionately about," says Blagg.

"The Centre for Cancer Therapeutics is a unique hybrid model bringing the best of academic research together with a drug discovery infrastructure and organization similar to many biotech companies. The people here are world-class and the recent successes such as the development of abiraterone and the discovery of small-molecule inhibitors of PI3 kinase demonstrate that this unique model can work."

Blagg began his career in industry after training in synthetic organic chemistry, receiving an M.A. and D.Phil. from Oxford University, UK, and completing a 2-year postdoc with Professor Wolfgang Oppolzer at the University of Geneva, Switzerland. "I applied for medicinal chemistry positions in the UK and Switzerland and was lucky enough to have offers to work with many of the big pharma companies at the time," recalls Blagg. "I joined Pfizer in the UK, which at the time was much smaller than it is now (number 14 in the world), because I felt it was the company where I would learn and be stretched the most - I liked the people I met at the interview and the way they approached their science. I was not

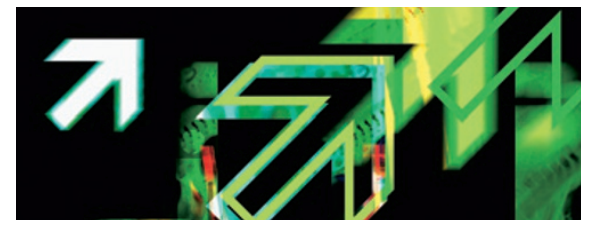

wrong; I never stopped learning, and I am proud to have spent 19 enjoyable years working there with great scientists and drug discoverers."

His medicinal chemistry experience over this time spanned therapeutic areas that included cardiovascular disease, urology, and inflammation, and he co-invented multiple clinical candidates. Spending a year at Pfizer's Groton labs in the US was particularly valuable. "It taught me so much about coping with change, different ways of doing science and different ways of working with people," says Blagg.

He feels that this experience has helped the transition to his latest position, where some of the most challenging aspects are getting up to speed with a new environment, new people, a new disease area and new projects. Most importantly though, he emphasizes the importance of collaboration. "For all the technological developments in drug discovery, success still comes down to choosing the right experiments, executing them well and learning from them - and this requires an open mind, innovative thinking, teamwork and dialogue," says Blagg. "Drug discovery is a team effort with exciting choices to make - that is the fun part."

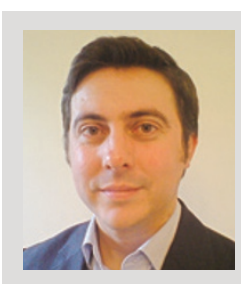

Andrew L. Hopkins, D.Phil.

SULSA Research Professor of Translational Biology and Chair of Medicinal Informatics, Division of Biological Chemistry and Drug Discovery, College of Life Sciences, University of Dundee, UK.

Last year, the Scottish Funding Council and six of Scotland's leading universities - Aberdeen, Dundee, Edinburgh, Glasgow, St Andrews and Strathclyde - announced the formation of the Scottish Universities Life Sciences Alliance (SULSA) with the aim of building on the region's strong reputation in life sciences. One of the first researchers from the pharma industry to join the new initiative is Andrew Hopkins, who was recently appointed as the SULSA Research Professor of Translational Biology and Chair of Medicinal Informatics at the University of Dundee.

"The combination of roles in translational biology and medicinal informatics provides a wonderful opportunity to develop a multidisciplinary programme to advance new methods in drug discovery in the public sector," says Hopkins. "As translational research rises up the policy agenda, and as the industry moves towards a network business model, licensing leads from a number of new sources, I believe it is in both the public and private sectors' interest to widen access to drug discovery tools and knowledge that have traditionally resided primarily in industry, such as the infrastructure and expertise for analysing compound structure-activity data."

Seeking to continue research in drug discovery following his D.Phil. in structural biology at Oxford University, UK, where he worked on the design of anti-HIV agents, the resources and expertise of a major pharma company were one of the factors that attracted Hopkins to join Pfizer. He applied for a protein crystallography position, but was offered a newly created position in informatics, initially focusing on target analysis. "I'm extremely grateful to the managers at Pfizer, in particular John Overington, for taking a chance on creating a position for me," says Hopkins. "Many organizations today recruit to specific job profiles rather than on the potential of the individual; there is a danger we may be losing some creative elements in a prescriptive approach."

During his 9 years at Pfizer, Hopkins continued to establish new functions based on the design and construction of major informatics systems, including founding an Indications Discovery Group, which he considers to be a particularly valuable experience. "The most important lesson I learnt was that with a common vision and trust, a team could achieve remarkable advances," says Hopkins. He and his colleagues also pioneered several widely recognized drug discovery concepts, such as the druggable genome and ligand efficiency.

He became aware of the new SULSA position at Dundee through a collaborator there - Michael Ferguson, Dean of the School of Research at the College of Life Sciences - who suggested that he should consider a move from industry to academia by applying. "I was attracted by its reputation as a pre-eminent centre for life sciences in Europe; its commitment to building a drug discovery unit; but most importantly, the opportunity to work with a number of great scientists,"says Hopkins. He is now finding the sense of freedom and creativity to be the most rewarding aspect of his new role. "The space to consider creative solutions to some of the more fundamental challenges in drug discovery and then the freedom to work with a wide range of scientists and organizations to realize that research is liberating." 